\title{
Preventive or restorative care for children?
}

\section{The influences on preventive care provided to children who frequently attend the UK General Dental Service}

\section{Tickle, K. M. Milsom, D. King and A. S. Blinkhorn Br Dent J 2003; 194: 329-332}

\section{Objectives}

To identify the relationship between the preventive and restorative care provided to children who frequently attend the General Dental Service in the UK after taking into account socio-economic status, gender and dental caries experience.

\section{Setting}

General dental practices in the North West of England.

Subjects and materials

The study design involved a retrospective investigation of case notes of 677 children who regularly attended 50 general dental practitioners. The complete history of the dental care received by each child during the primary dentition period was recorded. Analyses took place at the patient level. Information was recorded on the total number of carious teeth and restorative and preventive care provided to the children. Preventive care was categorised as dietary advice, oral hygiene instruction, prescription of fluoride tablets and application of fluoride varnish. Socio-economic status was measured using the Townsend score of the electoral ward of residence of each subject. Logistic regression models, taking into account the clustering of the subjects within dental practices were fitted to identify whether or not socio-economic status was significantly associated with provision of each category of preventive care, after controlling for gender, the total number of teeth affected by caries and the proportion of carious teeth which were restored.

\section{Results}

Children from poorer backgrounds were significantly more likely to receive oral hygiene instruction than their more affluent peers. Socio-economic status did not influence dentists' decisions to prescribe fluoride tablets, but children from affluent backgrounds were significantly more likely to have fluoride varnish applied to their teeth than children from deprived backgrounds after controlling for other factors. The more teeth affected by decay significantly increased the odds of giving dietary advice, prescription of fluoride tablets and application of fluoride varnish, but had no effect on whether or not oral hygiene instruction was given. As the percentage of decayed but filled teeth decreased the odds of giving dietary advice or applying fluoride varnish increased significantly.

\section{Conclusion}

It would appear that dentists are providing appropriate preventive care according to the aetiological causes of dental disease. They also look to be providing preventive care in compensation for decisions not to restore carious primary teeth. However the preventive care provided seems to be reactive to disease patterns, and in this high risk group of patients does not seem to be particularly effective.

\section{COMMENT}

The authors of this well written paper have previously reported on the fate of carious primary teeth of 677 children who regularly attended general dental practice in the UK. A total of 50 general dental practitioners participated. Only frequent attenders were included, frequent attenders being defined as those children attending the same dental practitioner at least every 18 months. Also, only children who had a history of interproximal caries in their primary molars were included. These children are particularly challenging patients for GDPs since, even though they attend regularly they are prone to caries. The aim of this aspect of the study was to ascertain the level and nature of preventive care provided.

The results of the earlier papers had indicated that many GDPs did not routinely restore primary molar teeth and if it was established that preventive care was not being provided for these patients then the charge of 'supervised neglect' could be justifiably made. In fact, the results do not support this charge since preventive care was found to be concentrated on those children with high levels of dental decay in their primary teeth. For example, the more teeth affected by decay significantly increased the odds of giving dietary advice, prescription of fluoride tablets and application of fluoride varnish, but no effect on whether or not oral hygiene instruction was given. There is no mention of the use of fissure sealants; it would be interesting to know if high levels of dental decay in primary molars was also a factor in the dentists decision to fissure seal first permanent molars.

Presumably the case notes studied would also have included details of this preventive strategy. It was somewhat surprising that advice on the appropriate use of fluoride toothpastes was rarely given. An important point made by the authors is that whilst the practitioners seems to be conscientious in their provision of preventive care for high risk children, this preventive care seems to be largely ineffective.

In general this study represents a further important contribution to the growing debate on what is the appropriate management of symptomless carious primary teeth. The widely held view that all such lesions should be restored, is being increasingly questioned. However, until such time as the alternative preventive approach is shown to be effective, it is difficult to adopt a general policy of non-restoration.

Denis O'Mullane, Director, Oral Health Services Research Centre, University Dental School and Hospital, Cork, Ireland.

\section{IN BRIEF}

- GDPs seem to be providing appropriate preventive care consistent with the aetiological causes of dental disease.

- Preventive care appears to be provided as an alternative to restorative care for some carious primary teeth.

- Preventive care in these high-risk children appears to be reactive to disease progression and is not particularly effective.

- There were widespread differences in the provision of preventive care amongst GDPs, this and the concerns about effectiveness point to a need for an evidence based and adequately funded prevention programme for GDPs to apply in their practices. 